where subscripts indicate partial differentiation, and where $e$ is chosen equal to \pm 1 so as to make $A$ positive. Differentiating $A$ with respect to $\theta$, and setting the result equal to zero, we get

(1) $F\left(-\phi_{x} \sin \theta+\phi_{y} \cos \theta\right)$

$$
-\left(\phi_{x} \cos \theta+\phi_{y} \sin \theta\right)\left(-F_{x^{\prime}} \sin \theta+F_{y^{\prime}} \cos \theta\right)=0,
$$

$F_{x^{\prime}}, F_{y^{\prime}}$ denoting partial derivatives of $F$ with respect to its third and fourth arguments respectively. Since

$$
F=F_{x^{\prime}} \cos \theta+F_{y^{\prime}} \sin \theta,^{*}
$$

equation (1) reduces to

(2) $\phi_{y}(x, y) F_{x^{\prime}}(x, y, \cos \theta, \sin \theta)$

$$
-\phi_{x}(x, y) F_{y^{\prime}}(x, y, \cos \theta, \sin \theta)=0,
$$

and if we define direction on the curve $\phi=c$ by means of the angle $\bar{\theta}=\arctan \left(-\phi_{x} / \phi_{y}\right)$, (2) becomes $F_{x^{\prime}}(x, y, \cos \theta, \sin \theta) \cos \bar{\theta}-F_{y^{\prime}}(x, y, \cos \theta, \sin \theta) \sin \bar{\theta}=0$.

But this equation determines the value of $\theta$ to which the curve $\phi=c$ is transversal. $\dagger$

Therefore the differential quotient $d \phi / d S$ is equal to zero in the direction tangent to the curve $\phi=c$ and has its maximum absolute value in the direction to which the curve $\phi=c$ is transversal.

WASHINGTON UNIVERSITY,

St. LouIs, Mo.

\title{
TANGENTIAL INTERPOLATION OF ORDINATES AMONG AREAS.
}

BY DR. C. H. FORSYTH.

(Read before the American Mathematical Society December 27, 1917.)

IF we wish to interpolate several values in each interval between the successive ordinates $u_{0}, u_{1}, u_{2}, \cdots, u_{n}$ by finite differences, only a low order of differences can with propriety be used, since high orders based on ordinary statistical data

* See Bolza, loc. cit., p. 196.

† See Bolza, loc. cit., p. 303. 
lead to errors. But low orders of differences mean that few of the ordinates can be used at a time, and hence the curve passing through the values intermediate to $u_{1}$ and $u_{2}$ is not in general identical with the curve passing through the values intermediate to $u_{2}$ and $u_{3}$, and similarly for the rest of the intervals. It follows that in such successive interpolations the final and complete series of interpolated values have discontinuities at their points of intersection $u_{1}, u_{2}$, etc., which are sometimes quite serious.

Osculatory interpolation represents an attempt to eliminate these discontinuities and succeeds for all practical purposes. It requires that whatever interpolation formula may be used as a basis, two intersecting interpolation curves passing through interpolated values in two adjacent intervals shall have the same slope and curvature at their point of intersection. Thus, the two curves are said to have a common osculating circle at this point and the modification of the basic interpolation formula is called the corresponding osculatoiy interpolation formula.

In recent years the most prominent statisticians have come to agree that differences higher than third or fouith are inappropriate for interpolating among ordinary statistical data and, as osculatory interpolation requires the use of fifth differences, formulas have been derived ignoring the idea of cuivvature and reducing the order required to third differences. However, in order to give full credit to the original plan even these third difference formulas are often referred to as "osculatory"; it has seemed more appropriate to the writer to refer to such formulas as "tangential" and the term will be used with that meaning in what follows.

In the December, 1916, issue of the Quarterly Publications of the American Statistical Association the writer gave the derivation of an interpolation formula which is to be usedexplained geometrically-for interpolating ordinates among areas. For example, it may be used to estimate the population for individual ages from populations given in age groups. The formula to second differences is as follows:

$$
\begin{aligned}
u_{x / t}=\frac{w_{0}}{t} & +(2 x-t+1) \frac{\Delta w_{0}}{t^{2} \cdot 2 !} \\
& +\left\{3 x^{2}+3 x(1-2 t)+\left(1-3 t+2 t^{2}\right)\right\} \frac{\Delta^{2} w_{0}}{t^{3} \cdot 3 !} .
\end{aligned}
$$


Thus, the individual value $u_{x / t}$ may be found from groups of $t$ individual values $-w_{0}, w_{1}, w_{2}$, etc.-and their differences, where

$$
w_{0}=u_{0 / t}+u_{1 / t}+\cdots+u_{(t-1) / t}
$$

and in general

$$
w_{x}=u_{x+0 / t}+u_{x+1 / t}+\cdots+u_{x+(t-1) / t} .
$$

The purpose of this paper is to modify formula (1) to give a "tangential" interpolation formula.

We assume the desired equation to be of the form

$$
u_{(t+x) / t}=u_{1}+a x+b x^{2}+c x^{3}+d x^{4},
$$

which evidently passes through the value $u_{1}$ for $x=0$.

For $x=t$

$$
u_{2}=u_{1}+a t+b t^{2}+c t^{3}+d t^{4} .
$$

Expressing $u_{1}$ and $u_{2}$ by formula (1), we have

(A) $u_{2}-u_{1}=a t+b t^{2}+c t^{3}+d t^{4}=\frac{\Delta w_{0}}{t}+(1+t) \frac{\Delta^{2} w_{0}}{2 t^{2}}$.

The derivative of (2) with respect to $x$ becomes

$$
D_{x} u_{(t+x) / t}=a+2 b x+3 c x^{2}+4 d x^{3},
$$

and for $x=0$

$$
D_{x} u_{1}=a .
$$

But from formula (1)

$$
D_{x} u_{x / t}=\frac{\Delta w_{0}}{t^{2}}+(2 x-2 t+1) \frac{\Delta^{2} w_{0}}{2 t^{3}},
$$

and for $x=t$

$$
D_{x} u_{1}=\frac{\Delta w_{0}}{t^{2}}+\frac{\Delta^{2} w_{0}}{2 t^{3}}
$$

Hence, by (4) and (6)

$$
a=\frac{\Delta w_{0}}{t^{2}}+\frac{\Delta^{2} w_{0}}{2 t^{3}}
$$


Formula (5) may be expressed also in terms of the differences of $u_{1}$; hence, as in (6)

$$
\begin{aligned}
D_{x} u_{2} & =\frac{\Delta w_{1}}{t^{2}}+\frac{\Delta^{2} w_{1}}{2 t^{3}} \\
& =\frac{\Delta w_{0}}{t^{2}}+(2 t+1) \frac{\Delta^{2} w_{0}}{2 t^{3}}+\frac{\Delta^{3} w_{0}}{2 t^{3}},
\end{aligned}
$$

since

and

$$
\Delta w_{1}=\Delta w_{0}+\Delta^{2} w_{0}
$$

$$
\Delta^{2} w_{1}=\Delta^{2} w_{0}+\Delta^{3} w_{0}
$$

Formula (3) for $x=t$ becomes

$$
D_{x} u_{2}=a+2 b t+3 c t^{2}+4 d t^{3} .
$$

Hence, from (7) and (8)

$$
\text { (C) } a+2 b t+3 c t^{2}+4 d t^{3}=\frac{\Delta w_{0}}{t^{2}}+(2 t+1) \frac{\Delta^{2} w_{0}}{2 t^{3}}+\frac{\Delta^{3} w_{0}}{2 t^{3}} \text {. }
$$

We now have three equations $(A),(B)$, and $(C)$ out of four necessary equations to determine the values of the four coefficients $a, b, c$, and $d$ in equation (2). Ordinarily, an equation of the third degree would be sufficient, in which case only three coefficients would be needed, but formula (1) is peculiar in the respect that when a group, say $w_{1}$, is broken up into the individual values $u_{1}, u_{1+1 / t}, \cdots u_{1+(t-1) / t}$ their sum must finally equal $w_{1}$ and in establishing formula (2) this requirement must be maintained. This requirement furnishes the desired fourth equation. If we give $x$ successively the values $0,1,2, \cdots, t-1$ in formula (2) and sum the results we obtain $w_{1}$ or $w_{0}+\Delta w_{0}$. This yields

$$
\begin{aligned}
w_{0}+\Delta w_{0}= & t u_{1}+\frac{t(t-1)}{2} a+\frac{t(t-1)(2 t-1)}{6} b \\
& +\frac{t^{2}(t-1)^{2}}{4} c+\frac{t(t-1)(2 t-1)\left(3 t^{2}-3 t-1\right)}{30} d .
\end{aligned}
$$

Transposing the term $t u_{1}$ to the left side and noting that by formula (1)

$$
u_{1}=\frac{w_{0}}{t}+(t+1) \frac{\Delta w_{0}}{2 t^{2}}+\left(1-t^{2}\right) \frac{\Delta^{2} w_{0}}{6 t^{3}}
$$


we have, on simplifying

$$
\text { (D) } \begin{array}{r}
a+\frac{(2 t-1)}{3} b+\frac{t(t-1)}{2} c+\frac{(2 t-1)\left(3 t^{2}-3 t-1\right)}{15} d \\
=\frac{\Delta w_{0}}{t^{2}}+(t+1) \frac{\Delta^{2} w_{0}}{3 t^{3}} .
\end{array}
$$

Solving the four equations $(A),(B),(C)$, and $(D)$ simultaneously, we obtain

$$
\begin{aligned}
& a=\frac{\Delta w_{0}}{t^{2}}+\frac{\Delta^{2} w_{0}}{2 t^{3}}, \\
& b=\frac{\Delta^{2} w_{0}}{2 t^{3}}+\frac{3 t^{2}-2}{4 t^{4}\left(t^{2}+1\right)} \Delta^{3} w_{0}, \\
& c=\frac{1-4 t^{2}}{2 t^{5}\left(t^{2}+1\right)} \Delta^{3} w_{0}, \\
& d=\frac{5 \Delta^{3} w_{0}}{4 t^{4}\left(t^{2}+1\right)} .
\end{aligned}
$$

Finally, substituting these values in equation (2) and collecting terms we obtain

$$
\begin{array}{r}
u_{(t+x) / t}=w+(2 x+t+1) \frac{\Delta w}{2}+\left(3 x^{2}+3 x+1-t^{2}\right) \frac{\Delta^{2} w}{6} \\
+x^{2}(x-t)\left(5 t x+2-3 t^{2}\right) \frac{\Delta^{3} w}{4 t\left(t^{2}+1\right)}
\end{array}
$$

where, for brevity, we let $\Delta^{n} w=\Delta^{n} w_{0} / t^{n+1}$ (and $w=w_{0} / t$ ).

In applying (9) to, say the group $w_{1}$, it is to be noticed that the slope at $u_{1}\left(=u_{t / t}\right)$ - by (6)-is determined by $w_{0}, w_{1}$, and $w_{2}$ (to be referred to for the present as "the first set") and at $u_{2}\left(=u_{2 t / t}\right)$ - by (8)-by $w_{1}, w_{2}$, and $w_{3}$ (to be referred to as "the second set"). Then, in applying the formula to the next group $w_{2}$, its first set (used to determine the slope at $u_{2}$ ) is identical with the second set of the preceding group and the interpolation curves of the two groups have the same slope at their point of intersection $u_{2}$; and similarly for $u_{3}, u_{4}$, etc. The idea of curvature could be added if desired in exactly the same way if higher differences were proper. 
When, as often happens, $t=5$, (9) becomes

$$
\begin{aligned}
u_{(5+x) / 5}=w+(x+3) \Delta w & +\left(x^{2}+x-8\right) \frac{\Delta^{2} w}{2} \\
& +x^{2}(x-5)(25 x-73) \frac{\Delta^{3} w}{(104) 5^{2}} .
\end{aligned}
$$

It is seldom desirable to use either (1) or (9) to determine a single individual value - they are generally used to break up a group of values completely. In the latter case, those familiar with the use of finite differences know that much labor can be saved by using the corresponding formulas for the leading term and differences of the individual values. The application of such formulas is much shorter on the average than the repeated application of either (1) or (9) and the majority of the computation consists in mere addition.

If we let $x$ equal successively $0,1, \cdots, t-1$ in formula (9) and difference the results four times we obtain the following leading term and differences

$$
\begin{aligned}
u_{1} & =w+\frac{t+1}{2} \Delta w+\frac{1-t^{2}}{6} \Delta^{2} w, \\
\delta u_{1} & =\Delta w+\Delta^{2} w+(1-t)\left(2+5 t-3 t^{2}\right) \frac{\Delta^{3} w}{4 t\left(t^{2}+1\right)}, \\
\delta^{2} u_{1} & =\Delta^{2} w+\left\{\left(2-3 t^{2}\right)(6-2 t)+5 t(14-6 t)\right\} \frac{\Delta^{3} w}{4 t\left(t^{2}+1\right)}, \\
\delta^{3} u_{1} & =\left\{6\left(2-3 t^{2}\right)+30 t(6-t)\right\} \frac{\Delta^{3} w}{4 t\left(t^{2}+1\right)}, \\
\delta^{+} u_{\perp} & =t 5 ! \Delta^{3} w,
\end{aligned}
$$

where, it is to be remembered, $\Delta^{n} w=\Delta^{n} w_{0} / t^{n+1}$ (and $\left.w=w_{0} / t\right)$.

When $t$ has the value 5 the third differences prove relatively difficult to compute owing to the presence of a " 13 " in the denominator. If in the difference $\delta u_{1}, 2.7 \Delta^{3} w / 13$ is replaced by $2.6 \Delta^{3} w / 13$ and in all the rest $\Delta^{3} w / 13$ is replaced by $\Delta^{3} w / 12$ the leading term and differences (for $t=5$ ) become

$$
\begin{array}{rlr}
u_{1} & =w+3 \Delta w-4 \Delta^{2} w \\
\delta u_{1} & = & \Delta w+\Delta^{2} w+.2 \Delta^{3} w, \\
\delta^{2} u_{1} & = & \Delta^{2} w-.2 \Delta^{3} w, \\
\delta^{3} u_{1} & = & -.6 \Delta^{3} w \\
\delta^{4} u_{1} & = & +.002 \Delta^{3} w_{0},
\end{array}
$$


where, it is to be remembered, $\Delta^{n} w=\Delta^{n} w_{0} / 5^{n+1}$ (and $w=w_{0} / 5$ ). It should be noticed, however, that $\delta^{4} u_{1}$ is expressed more simply by $.002 \Delta^{3} w_{0}$.

The arbitrary changes just suggested give an error of only about one half of one per cent of $\Delta^{3} w_{0}$ in $w_{1}$ (the sum of the individual values interpolated). The error in each individual value would then be much less and if only two more decimal places are used than are to be finally retained-which are all that are ordinarily necessary as found by experience-the errors would not appear at all in the results. As a check upon the work the sum of the individual values interpolated should be $w_{1}$ as given originally.

It should be pointed out that the formulas derived above can not be used for "end" values; that is, if the groups of values were $w_{0}, w_{1}, w_{2}, \cdots, w_{n}$ the formulas could not be used for interpolating or breaking up $w_{0}$ or $w_{n}$, for the derivation of the formulas is based upon the use of four values $\left(w_{0}, w_{1}, w_{2}\right.$, and $w_{3}$ ) to break up $w_{1}$; that is, there must always be at least one group preceding the group to be broken up. To break up "end" values formula (1) could be used or the formulas for the leading term and differences to be found in the article cited.

Dartmouth College,

HaNover, N. H.

\section{NON-EUCLIDEAN GEOMETRY.}

Geometrical Researches on the Theory of Parallels. By N. Lobachevski. Translated from the original by G. B. Halsted. New edition. Chicago and London, Open Court, 1914. 8vo. 50 pages. Cloth, price $\$ 1.25$.

NoN-EUCLIDEAN geometry had two independent discoverers: Johann Bolyai (1802-1860), a Hungarian officer in the Austrian army; and Nicolaus Lobachevsky (1793-1856), son of a Russian peasant, and graduate, professor, and rector, of the University of Kasan.*

As early as 1823 the former had grasped the real nature of his problem, and in 1829 he sent a completed manuscript on the subject to his father, Wolfgang Bolyai, who was a

* The best history of non-euclidean geometry is by Bonola. It was translated into English and edited by H. S. Carslaw (Chicago, 1912). 